\title{
Invited Discussion on: Post-Operative Complications and Patient Satisfaction After Abdominal Etching-Prospective Case Series of 25 Patients
}

\author{
Abdominal Definition Liposuction Or High Definition Liposculpture?
}

\author{
Alfredo E. Hoyos ${ }^{1}$ (D) Mauricio E. Perez ${ }^{2}$
}

Received: 5 February 2020/Accepted: 12 February 2020/Published online: 10 March 2020

(C) Springer Science+Business Media, LLC, part of Springer Nature and International Society of Aesthetic Plastic Surgery 2020

Level of Evidence $V$ This journal requires that authors assign a level of evidence to each article. For a full description of these Evidence-Based Medicine ratings, please refer to the Table of Contents or the online Instructions to Authors www.springer.com/00266.

\section{Background}

Body contouring surgery has constantly evolved with great improvements described in recent decades. Since Illouz [1] described liposuction as a secure technique for fat removal, several variations to the original technique have been reported, in order to make it safer and easier to perform for both the patient and the surgeon. One of these great improvements has been the introduction of artistic anatomy and superficial framing as fundamental premises for high definition liposculpture [2]. On the other side, the incorporation of new technologies, such as VASER (vibration amplification of sound energy at resonance), MicroAire (power-assisted liposuction), J-Plasma (helium-based plasma), among others, has substantially eased the process of body contour surgery. These devices, particularly VASER, have demonstrated lower complication rates compared to those of standard liposuction, in addition to high satisfaction indexes among patients [3].

Liposuction still remains as the top two procedures worldwide, below breast augmentation [4]; however, the percentage of liposuction procedures has been increasing

\footnotetext{
Alfredo E. Hoyos

alhoyos@gmail.com

1 Bogota, Colombia

2 New York, USA
}

compared to that of breast augmentation since 2014. For this reason, recent research has focused on liposuction safety and lasting results. Some studies have detailed the most common complications associated with VASER-assisted liposculpture compared to standard liposuction; a clear difference in safety has pointed to the use of ultrasound in order to lower the risk of ischemia and necrosis over the superficial skin flap, as well as less bleeding [5], less trauma, and consequently faster recovery [6].

\section{Carving Superficial Anatomy}

Since the advent of third-generation ultrasound devices, the authors described the high definition liposculpture (HDL) [6] and a new concept was brought in to change the surgeon's perspective: The knowledge of human anatomy switched from a medical to an artistic and metaphoric approach such as sculptors and artists do [2]. As a result, we started working over the superficial fat layer to perform a more precise and aesthetic procedure by exposing the normal underlying muscular anatomy and histology. The concepts of lights and shadows allowed the surgeon to shape a muscular contour, and results became natural for both the slim and the overweight patient, since many muscle features were acknowledged: projection, depth, surroundings and dynamics [2,7]. The latter was described as an improvement in the initial 3D approach, considering that some muscles have great changes over the surface anatomy during active contraction compared to those in the resting position [8]. This allowed us to further improve the natural appearance of our results, by taking into account the movement of some muscles such as the pectorals, deltoids, triceps, biceps and calves. Consequently, these new ideas encouraged surgeons to consider other 
challenges related to the procedure itself: indications, limits, contraindications and complications.

\section{Limited Abdominal Definition Surgery}

The involvement of the superficial fat layer was something not considered before HDL. Its specific treatment and framing revolutionized body sculpting procedures; however, some important concepts and clarifications have to be done:

1. Most patients requesting for high definition liposculpture are not usually fit or athletic. In fact, the vast majority are overweight or at the top of the normal body mass index (BMI). Then, selective bias most commonly happens in studies that enroll patients that are already athletic or in a normal weight range.

2. One of the more common mistakes in body contour surgery is the limitation of high definition liposculpture (HDL) to a unique muscle. As mentioned before, it was invented as a whole-body procedure [6] or at least performed over a complete body segment: upper [8] and lower limbs, abdomen and torso [7], which allows the surgeon to achieve long-lasting natural and authentic outcomes. The "unique-muscle" HDL surgery should not exist as the artistic anatomy premises-harmony, positive and negative spaces, muscle contours and dynamics—-would be directly violated [2].

3. We do not recommend to perform deep and superficial framing limited to the rectus abdominis tendinous insertions, linea alba and/or lateral borders: If a deep fat layer remained over the rectus abdominis muscle fibers, then asymmetries and unnatural results may be present in the short- and long-term follow-up, which could be even more perceptible if the patient were to gain weight ("Fat fit man").

4. In our experience, the aid of third-generation ultrasound has improved the surgeons' performance during the procedure and has decreased the risk for complications, since HLD requires large-volume infiltration and liposuction. However, the device cost versus effectiveness comparison is relatively difficult to measure, due to the fact that HDL technique actually includes the use of a third-generation ultrasound device.

5. Muscles with large muscle mass benefit from the fat grating, since its vascularization and power allow them to put up some additional volume when needed. Fat grafting improves muscle projection and enhances its athletic appearance, but it is not recommended for small muscles such as the rectus abdominis, serratus and forearm muscles.

6. Compression garments are essential in the post-operative HDL outcomes. Gentle but constant compression must be achieved for the next 4-6 weeks following the procedure with foam vests [2]. Skin adhesion and healing occur once the acute inflammation phase has concluded and the regenerative tissue appears. Inasmuch as the superficial layer thickness is decreased, firm or excess compression must be avoided in order to allow acute phase physiologic inflammation and reperfusion; otherwise, the risk of flap ischemia and necrosis could increase. In effect, we do not recommend the use of firm materials or garments to "forcefully" shape the abdominal muscles and also avoid placing any material that could potentially injure the superficial skin layers (tape, silicone bands, micropore, etc).

\section{Conclusion}

High definition liposculpture (HDL) should not be compared to traditional liposuction, since HDL is a 360-degree procedure involving multiple muscle groups and regions [2]. Carving those structures will enhance and create an athletic and muscular appearance in men and a slim and natural looking in women. In contrast, traditional liposuction is focused on gross fat resection and does not allow the surgeons to recreate the underlying muscle anatomy. Technology might not be seen as an obstacle for the surgeon, since various studies have reported benefits in its use for cosmetic and specifically body contour surgery. Still, further studies need to be performed to analyze the proper cost-effectiveness of the third-generation ultrasound in high definition liposculpture. On the other side, new surgical techniques must be suitable for the whole population, or the great majority, rather than selective for specific individuals. HDL has been reported in multiple types of patients and populations, regarding some anatomical areas, but further research is required to support its use in different body types, including patients with different anatomical features as well as requesting different grades of muscle definition.

Finally, we recommend keeping always in mind the basic premises of high definition liposculpture in order to achieve the best results possible. Our experience has allowed us to correct and polish the different aspects of dynamic and high definition liposculpture, improving our practice on a daily basis. 
Funding The authors received no financial support for the research, authorship and publication of this article.

\section{Compliance with Ethical Standards}

Conflict of interest Dr. Hoyos was an unpaid consultant and speaker for the product development team of Sound Surgical Technologies (SST) system and Cannulas (now: VASER $\odot 2018$ Solta MedicalBausch Health Companies Inc.) up to May 2013. He receives royalties for the liposuction kits named after him. The authors declare that they have no conflicts of interest to disclose.

Ethical Approval This article does not contain any studies with human participants or animals performed by any of the authors.

Informed Consent For this type of study, informed consent is not required.

\section{References}

1. Illouz YG (1989) Body sculpturing by lipoplasty (English edition). Churchill-Livingstone, London
2. Hoyos AE, Prendergast PM (2014) High definition body sculpting: art and advanced lipoplasty techniques. Springer, Heidelberg

3. Rohrich RJ, Beran SJ, Kenkel JM (1998) Ultrasound-assisted liposuction, 1st edn. Thieme Medical Publishers, Inc., St Louis

4. Plasticsurgery.org (2019) https://www.plasticsurgery.org/ documents/News/Statistics/2018/plastic-surgery-statistics-fullreport-2018.pdf. Accessed 4 Feb 2020

5. Garcia O (2010) Comparison of blood loss in suction-assisted lipoplasty and third-generation ultrasound-assisted lipoplasty. In: Shiffman M, Di Giuseppe A (eds) Body contouring. Springer, Berlin, Heidelberg, pp 565-573

6. Hoyos AE, Millard JA (2007) VASER-assisted high-definition liposculpture. Aesthet Surg J 27(6):594-604

7. Hoyos A, Perez M (2012) Dynamic-definition male pectoral reshaping and enhancement in slim, athletic, obese, and gynecomastic patients through selective fat removal and grafting. Aesthet Plastic Surg 36(5):1066-1077

8. Hoyos A, Perez M (2012) Arm dynamic definition by liposculpture and fat grafting. Aesthet Surg J 32(8):974-987

Publisher's Note Springer Nature remains neutral with regard to jurisdictional claims in published maps and institutional affiliations. 